\title{
Layer antiferromagnetic state in bilayer graphene: A first-principles investigation
}

\author{
Yong Wang, ${ }^{1}$ Hao Wang, ${ }^{1}$ Jin-Hua Gao, ${ }^{2,1, *}$ and Fu-Chun Zhang ${ }^{1,3}$ \\ ${ }^{1}$ Department of Physics, The University of Hong Kong, Hong Kong SAR, China \\ ${ }^{2}$ Department of Physics, Huazhong University of Science and Technology, Wuhan, Hubei, China \\ ${ }^{3}$ Department of Physics, Zhejiang University, Hangzhou, China \\ (Received 21 January 2013; revised manuscript received 12 April 2013; published 9 May 2013)
}

\begin{abstract}
The ground state of bilayer graphene is investigated using density functional calculations with a local spindensity approximation. We find a ground state with layer antiferromagnetic ordering, which is one of the candidate states suggested by former studies based on a simplified model. The calculations prove that the layer antiferromagnetic state is stable even if the nonlocal Coulomb interaction and the remote hopping are included. The gap of the correlated ground state is about $1.8 \mathrm{meV}$, in reasonable agreement with the experimental value. The surface magnetism in the bilayer graphene is of the order of $10^{-2} \mu_{B} / \mathrm{nm}^{2}$.
\end{abstract}

DOI: 10.1103/PhysRevB.87.195413

PACS number(s): 73.22.Pr, 71.30.+h, 73.21.Ac, 75.70.Cn

\section{INTRODUCTION}

Graphene, the star material currently, has attracted great attention and stimulated intensive research since its birth. ${ }^{1}$ Many exotic physical properties of graphene, including the anomalous integer quantum Hall effect, Klein tunneling, etc., have been revealed, which promises huge potentials in practical applications..$^{2-5}$ However, one major roadblock for the practical applications of graphene in electronics is the small on/off ratio due to its gapless ground state. One alternative way to overcome the difficulty is to exploit the AB-stacked bilayer graphene (BLG) instead, where a gap can be formed and tuned by chemical doping or the external electric field. ${ }^{6,7}$ For example, unipolar transport has been demonstrated in a field-effect transistor based on $\mathrm{BLG},{ }^{8}$ and high-frequency manipulation of the BLG quantum dot has been realized. ${ }^{9}$ Recently, the results of several experiments on the ultraclean suspended BLG suggest that an intrinsic gap may exist at the charge neutrality point, which is attributed to the formation of certain ordered ground states due to spontaneously symmetry breaking. ${ }^{10-15}$ This finding has triggered the interest to study many-body physics near the Dirac point in graphene materials, which may provide not only new insight into the effects of electron correlations but also a new possibility to manipulate the energy gap for practical applications. At the moment, the nature of the correlated ground state of BLG is still unclear and highly debated, and different candidate states have been proposed theoretically. These include the layer antiferromagnetic (LAF) state, ${ }^{16-21}$ pseudospin magnetism, ${ }^{22}$ the quantum anomalous Hall state, ${ }^{23}$ the quantum spin Hall state, ${ }^{18,24}$ and a gapless nematic state. ${ }^{18,25-27}$

The existing debates are mainly due to the facts that only a few transport measurements are available so far ${ }^{10-15}$ and that the effective parameters of BLG used in model studies are not given a priori but are fitted to the experimental results. ${ }^{16,17,22-28}$ Another way to get these input parameters in the effective model is from the ab initio calculation results for graphene and graphite systems. ${ }^{29}$ However, the value of the energy gap obtained in this way is two orders of magnitude larger than the one observed in experiments. ${ }^{19}$ Thus, a satisfactory interpretation of the observed energy gap in ultraclean BLG with no empirical parameter is still lacking, not to mention the other observable physical quantities. Further, the simple Hubbard model for
BLG usually only includes the local Coulomb interaction, and two important factors are ignored. One is the influence of nonlocal components of the Coulomb interaction beyond the Hubbard $U$ term. Although these terms are smaller than the on-site Coulomb interaction, some recent theoretical works indicate that these terms do affect the correlated ground state of BLG. ${ }^{29}$ The other is the effect of the remote hopping terms, which essentially modifies the parabolic feature of the energy bands near the Dirac points. The first-principles calculation based on density functional theory (DFT) has the advantages that no empirical fitting parameter is needed and the secondary effects, such as the nonlocal Coulomb interaction and remote hopping, are naturally included. The first-principle method has been successfully used to study the edge magnetization in graphene nanoribbons ${ }^{30,31}$ and surface magnetization in eight-layer graphite. ${ }^{32,33}$ In this paper, we perform the DFT calculations to study the ground state of BLG, which gives a reasonable energy gap of the ground state compared with the experimental value. Further analysis of the calculated results shows that the correlated ground state of BLG is a spin-density-wave-like state, where the spin ordering is the same as the LAF state revealed in the former model studies. The calculations give the surface magnetism of the LAF state in BLG, which can be tested in further experiments. Due to the merits of the firsts-principle method, our calculation here is quantitative and thus is distinct from the former model studies. It is helpful to identify the nature of the correlated ground state in BLG and further material applications.

\section{RESULTS AND DISCUSSION}

The DFT calculations of the electron structures for BLG are performed with the ABINIT software package. ${ }^{34}$ The slab model consisting of the two graphene layers and a vacuum layer is constructed for the calculations with periodic boundary conditions. There are four carbon atoms in the primitive cell of the AB-stacked BLG, and the geometry structure of a $2 \times 2$ supercell is shown in Fig. 1(a). In each layer, there are two sublattices, i.e., sublattices $A$ and $B$. We use the experimental value of $3.35 \AA$ for the layer separation since the van der Waals interactions cannot be captured by local-density approximation (LDA). ${ }^{35}$ A $23.4-\AA \AA$ vaccum layer, which is seven times the layer separation, is thick enough to avoid the 


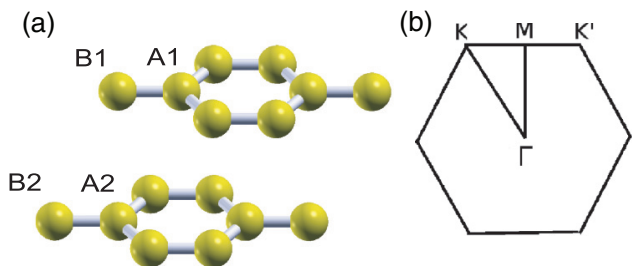

FIG. 1. (Color online) (a) The geometry structure of a $2 \times 2$ supercell of BLG. The four carbon atoms in the primitive cell are denoted as A1, B1, A2, and B2, respectively. (b) Brillouin zone (BZ) of BLG. $\Gamma, M, K$, and $K^{\prime}$ denote the high-symmetry points in the BZ.

intercell interactions between the graphene layers. The cutoff energy for the plane-wave basis set is chosen as $40 \mathrm{Ha}$, and the Troullier-Martins (TM) norm-conserving pseudopotential for the carbon element is exploited. The $k$ points for sampling the Brillouin zone (BZ) are generated by a $60 \times 60$ MonkhorstPack (MP) grid. In order to investigate the possible spin order, the local spin-density approximation (LSDA) is exploited in the exchange-correlation functional, and the convergence criteria for the total energy difference is $10^{-10} \mathrm{Ha}$.

The band structures and density of states (DOS) of BLG from the DFT + LSDA calculations are shown in Figs. 2(a) and 2(b), respectively. A fine band structure around Dirac point $K$ is shown in Fig. 2(c), where a minor band gap is opened around Dirac point $K$. The calculated band gap $E_{\text {gap }} \approx 1.8 \mathrm{meV}$ is comparable to the experimental values of $2-3 \mathrm{meV},{ }^{13-15}$ considering that LSDA calculations usually underestimate the band gap. For comparison, we also performed the DFT + LDA calculation without including the spin degrees of freedom, where no energy gap is found in this case, which is the same as in the previous studies. ${ }^{36}$ As shown below, the opening gap originates from the LAF ground state, and the results here suggest that the LAF state is still robust in the presence of nonlocal Coulomb interaction, at least at the level of LDA. (a)

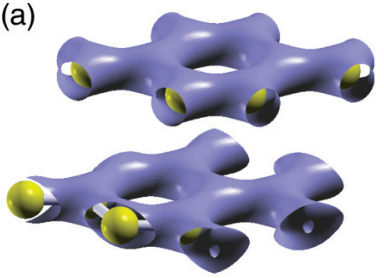

(b)

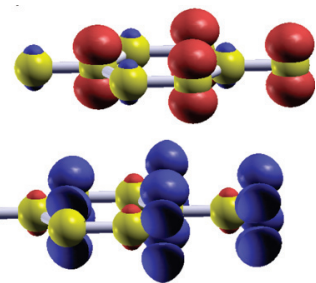

FIG. 3. (Color online) (a) Isosurface of the charge distribution with an isovalue of $1 \AA^{-3}$. (b) Isosurface of the spin polarization distribution with an isovalue of $1.7 \times 10^{-4} \AA^{-3}$ (red) and $-1.7 \times$ $10^{-4} \AA^{-3}$ (blue).

In BLG, the low-energy spectrum near the Dirac point is modified by the interlayer hopping between atom sites $\mathrm{A} 1$ and $\mathrm{B} 2$, where the isoenergetic lines around the highsymmetry point $K\left(K^{\prime}\right)$ are split into four pockets with one central part at $K\left(K^{\prime}\right)$ and three leg parts on the $K-\Gamma$ $\left(K^{\prime}-\Gamma\right)$ symmetry lines. ${ }^{2,37}$ This trigonal warping effect has a prominent effect on the physical properties of BLG, such as the weak localization ${ }^{38}$ and minimal conductivity. ${ }^{39}$ For the correlated ground states considered here, the presence of trigonal warping may invalidate the discussions based on the massive-chiral band mode ${ }^{17}$ or suppress the flow of couplings in the renormalization group treatment. ${ }^{27}$ Our first-principles calculation show that the LAF state is still stable in the presence of the trigonal warping effect. The energy-gap function near the high-symmetry point $K$ is given in Fig. 2(d), where the minimal gap is about $1.8 \mathrm{meV}$, and the threefold rotational symmetry due to the trigonal warping is shown.

The ground state of BLG as the LAF state in the DFT calculations is identified by analyzing its charge density and spin-polarization distributions. The isosurface of the charge density is illustrated in Fig. 3(a), which is basically extended along the $\mathrm{C}-\mathrm{C}$ bonds in each layer. We estimate the charge on each atom using the Hirshfeld method. ${ }^{40}$ Calculations with LDA and LSDA give the same charge distributions.
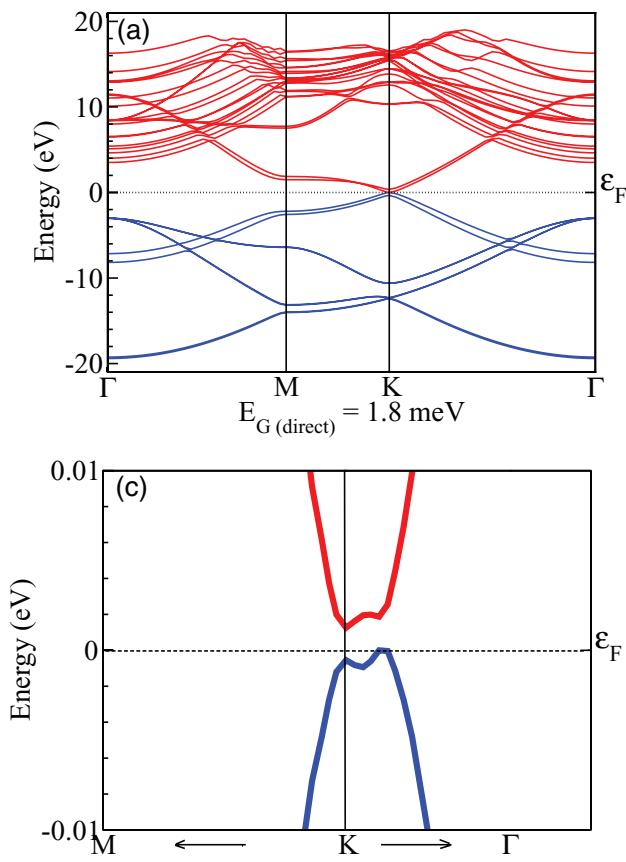
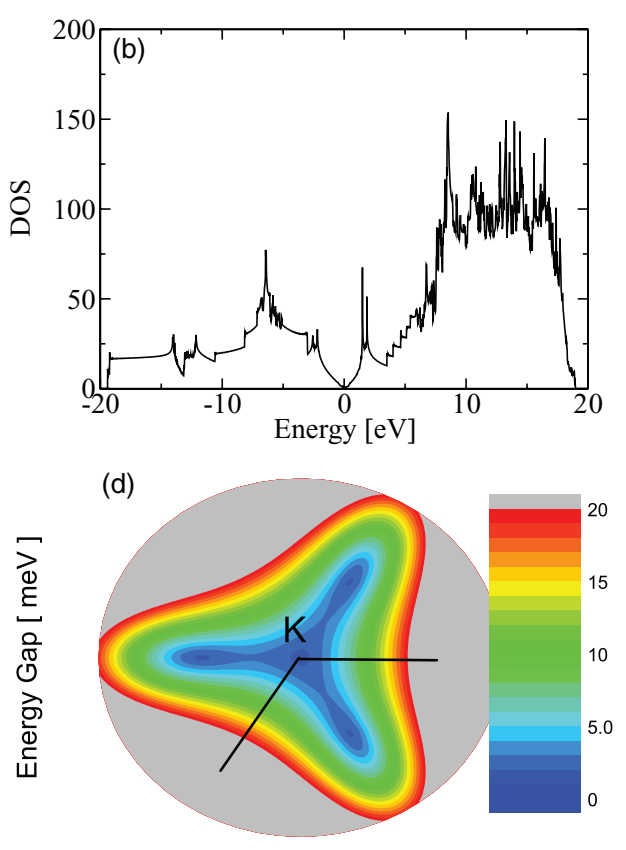

FIG. 2. (Color online) (a) Band structure of BLG from DFT + LSDA calculations. (b) DOS of BLG from DFT + LSDA calculations. (c) Fine band structure of BLG around Dirac point $K$ from DFT + LSDA calculations. (d) Trigonal warping effect on the gap function near the high-symmetry point $\mathrm{K}$. 
(a)

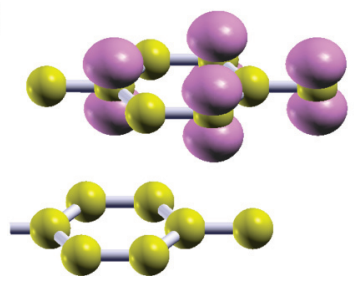

(b)

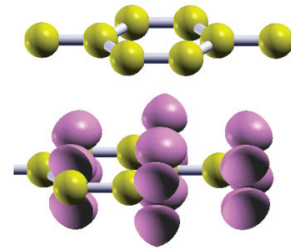

FIG. 4. (Color online) Isosurfaces of the norm of the wave function (spin up) for states at the $K$ point. (a) The state on the valence band; (b) the state on the conductance band. The isovalue is $0.2 \AA^{-3}$, and the wave function has been normalized in one primitive cell.

This implies that the energy gap in the LSDA calculation has nothing to do with the charge redistribution. For the spin distribution, the LDA results with LDA give no spin ordering because the spin degree of freedom is ignored, while the LSDA results give the layer antiferromagnetic order. As shown in Fig. 3(b), the spin polarization is mainly localized around the A1 and B2 atoms. The Hirshfeld method gives the spin polarization around the A1 atom as about $5.5 \times 10^{-4}$ and that around B2 as $-5.5 \times 10^{-4}$. Note that "spin polarization" here is defined as $n_{\uparrow}-n_{\downarrow}$, where $n_{\uparrow}\left(n_{\downarrow}\right)$ is the charge number with up spin (down spin). For the A2 and $\mathrm{B} 1$ atoms, the spin polarization is very tiny and at least one order of magnitude smaller than the spin polarization of the A1 and B2 atoms. Therefore, the net spin in each layer is nonzero, but that of the whole system is zero. This is just the layer antiferromagnetic state predicted by former mean-field studies. In Ref. 41, a self-consistent mean-field calculation gives similar spin ordering, and the largest spin polarization on one atom is of the order of $10^{-4}$ by fitting to an experimental value of the gap of $2 \mathrm{meV}$. Thus the first-principles results here are semiquantitatively in agreement with the mean-field studies, which provide essential support to the latter by including the influence of the nonlocal Coulomb interaction and remote hopping. Further, the DFT calculations give the surface magnetism as about $10^{-2} \mu_{B} / \mathrm{nm}^{2}$, which can be directly detected by spin-polarized scanning tunneling microscopy. The surface magnetism has been reported in trilayer and eight-layer graphene systems. ${ }^{32,33,42}$

The LAF state is further investigated from the peculiar wave functions near the Dirac points. When the electronelectron interaction is not considered, the low-energy states of BLG have layer symmetry, spin symmetry, and valley symmetry. ${ }^{22}$ The inclusion of electron-electron interaction can spontaneously break some symmetry and induce several possible correlated ground states. ${ }^{43}$ For the LAF state, the two layers have opposite spin polarization, and the layer symmetry is broken for each spin direction. For the up spin, the states on the conductance band near the Fermi level are localized on one layer, and the states on the valence band are on the other layer. However, for the down spin, the layer dependence is inverted. These features of the wave functions of the LAF state are demonstrated in the mean-field studies based on a simplified model $^{41}$ and also in the first-principles results here. Taking one spin direction, for example (say the up spin), we plot the wave functions for both conductance and valence bands at the $K$ point in Figs. 4(a) and 4(b), respectively. As we expected, the state on the valence (conductance) band is on the top (bottom) layer. However, the wave function for the states a small distance away from the Fermi level does not have such spin- and layer-dependent distribution, and the wave function spread uniformly among the two layers instead. The results here confirm that the electron-electron interaction in the BLG system only influences the low-energy states very close to the Fermi level, and the spin distribution of the LAF state is contributed by these states having spin ordering.

As discussed above, the DFT calculations gives a LAF ground state with an energy gap of about $1.8 \mathrm{meV}$. Compared with former model studies, the description of the LAF ground state here is more quantitative and realistic. One reason is that all the secondary effects, such as the nonlocal Coulomb interactions and the remote-hopping-induced trigonal warping, are included in the first-principles calculations. The other is that the predicted energy gap is consistent with the experimental value and is the most convincing quantitative result so far. Therefore, our work provides useful information for further studies of the LAF ground state in BLG. For example, we give the order of magnitude of surface magnetization of the LAF state, which is actually a key quantity for the LAF state. Considering that there is still no final consensus on the nature of the correlated ground state of BLG at the moment, the possible candidates have to be experimentally probed via detecting their characteristic properties, not only the energy gap. For the LAF state, detecting its surface magnetism can give evidence of its existence. The calculated surface magnetism here is about $10^{-2} \mu_{B} / \mathrm{nm}^{2}$, which offers a criterion for the experimental design.

Finally, we argue that the on-site Coulomb interaction in single-layer graphene and bilayer graphene are different, which may be the reason why former model studies give a much larger energy gap. In a recent quantum Monte Carlo calculation, the energy gap of the LAF state in BLG was estimated to be $200 \mathrm{meV}$ with the value of on-site Coulomb interaction of the single layer graphene derived from the firstprinciples calculations. ${ }^{19,29}$ Based on our calculation results here, it is reasonable to guess that the value of the Coulomb interaction of single-layer graphene is not suitable for this problem. The on-site Coulomb interaction may be different in the single-layer and bilayer graphene because of their different energy bands. Note that the on-site Coulomb interaction in BLG is actually in the region where the energy gap is quite sensitive to the value of interaction. ${ }^{41}$ A small variation of the interaction strength will dramatically change the energy gap. In addition, the other effects, e.g., nonlocal Coulomb interaction and the remote hopping, can also influence the final results because the energy band near the Dirac point is modified.

\section{CONCLUSION}

In summary, first-principles calculations have been performed to investigate the ground state of BLG. The LAF ground state is found in the LSDA calculations, and a reasonable energy gap compared with the experimental results is obtained. The results support the fact that the LAF state in $\mathrm{BLG}$ is robust in the presence of nonlocal Coulomb interaction and remote hopping. More detailed information on the LAF state is also given by the first-principles calculations, where the 
largest spin polarization on one atom is about $5.5 \times 10^{-4}$ and the surface magnetism is of the order of $10^{-2} \mu_{B} / \mathrm{nm}^{2}$. Our results here give a quantitative description of the LAF state in the BLG system, which is useful for further experimental investigations and practical applications.
We acknowledge some of financial support from HK-SAR RGC Grant No. HKU 701010, CRF Grant No. HKU 707010, and the Hong Kong University Grant Council (Grant No. AoE/P-04/08). J.H.G. is supported by the National Natural Science Foundation of China (Project No. 11274129). *jinhua@ @ust.edu.cn

${ }^{1}$ A. K. Geim and K. S. Novoselov, Nat. Mater. 6, 183 (2007).

${ }^{2}$ A. H. Castro Neto, F. Guinea, N. M. R. Peres, K. S. Novoselov, and A. K. Geim, Rev. Mod. Phys. 81, 109 (2009).

${ }^{3}$ S. Das Sarma, S. Adam, E. H. Hwang, and E. Rossi, Rev. Mod. Phys. 83, 407 (2011).

${ }^{4}$ M. O. Goerbig, Rev. Mod. Phys. 83, 1193 (2011).

${ }^{5}$ V. N. Kotov, B. Uchoa, V. M. Pereira, F. Guinea, and A. H. C. Neto, Rev. Mod. Phys. 84, 1067 (2012).

${ }^{6}$ T. Ohta, A. Bostwick, T. Seyller, K. Horn, and E. Rotenberg, Science 313, 951 (2006).

${ }^{7}$ Y. Zhang, T.-T. Tang, C. Girit, Z. Hao, M. C. Martin, A. Zettl, M. F. Crommie, Y. R. Shen, and F. Wang, Nature (London) 459, 820 (2009).

${ }^{8}$ H. Miyazaki, S.-L. Li, S. Nakaharai, and K. Tsukagoshi, Appl. Phys. Lett. 100, 163115 (2012).

${ }^{9}$ S. Droscher, J. Guttinger, T. Mathis, B. Batlogg, T. Ihn, and K. Ensslin, Appl. Phys. Lett. 101, 043107 (2012).

${ }^{10}$ B. E. Feldman, J. Martin, and A. Yacoby, Nat. Phys. 5, 889 (2009).

${ }^{11}$ R. T. Weitz, M. T. Allen, B. E. Feldman, J. Martin, and A. Yacoby, Science 330, 812 (2010).

${ }^{12}$ A. S. Mayorov, D. C. Elias, M. Mucha-Kruczynski, R. V. Gorbachev, T. Tudorovskiy, A. Zhukov, S. V. Morozov, M. I. Katsnelson, V. I. Falko, A. K. Geim, and K. S. Novoselov, Science 333, 860 (2011).

${ }^{13}$ J. Velasco, Jr., L. Jing, W. Bao, Y. Lee, P. Kratz, V. Aji, M. Bockrath, C. N. Lau, C. Varma, R. Stillwell, D. Smirnov, F. Zhang, J. Jung, and A. H. MacDonald, Nat. Nanotechnol. 7, 156 (2012).

${ }^{14}$ W. Bao, J. Velasco, Jr., F. Zhang, L. Jing, B. Standley, D. Smirnov, M. Bockrath, A. H. MacDonald, and C. N. Lau, Proc. Natl. Acad. Sci. USA 109, 10802 (2012).

${ }^{15}$ F. Freitag, J. Trbovic, M. Weiss, and C. Schönenberger, Phys. Rev. Lett. 108, 076602 (2012).

${ }^{16}$ J. Nilsson, A. H. Castro Neto, N. M. R. Peres, and F. Guinea, Phys. Rev. B 73, 214418 (2006).

${ }^{17}$ F. Zhang, H. Min, M. Polini, and A. H. MacDonald, Phys. Rev. B 81, 041402(R) (2010).

${ }^{18}$ Y. Lemonik, I. L. Aleiner, and V. I. Falko, Phys. Rev. B 85, 245451 (2012).

${ }^{19}$ T. C. Lang, Z. Y. Meng, M. M. Scherer, S. Uebelacker, F. F. Assaad, A. Muramatsu, C. Honerkamp, and S. Wessel, Phys. Rev. Lett. 109, 126402 (2012).
${ }^{20}$ M. M. Scherer, S. Uebelacker, and C. Honerkamp, Phys. Rev. B 85 , 235408 (2012).

${ }^{21}$ M. Kharitonov, Phys. Rev. B 86, 195435 (2012).

${ }^{22}$ H. Min, G. Borghi, M. Polini, and A. H. MacDonald, Phys. Rev. B 77, 041407 (2008).

${ }^{23}$ R. Nandkishore and L. Levitov, Phys. Rev. B 82, 115124 (2010).

${ }^{24}$ F. Zhang, J. Jung, G. A. Fiete, Q. Niu, and A. H. MacDonald, Phys. Rev. Lett. 106, 156801 (2011).

${ }^{25}$ O. Vafek and K. Yang, Phys. Rev. B 81, 041401(R) (2010).

${ }^{26}$ Y. Lemonik, I. L. Aleiner, C. Toke, and V. I. Falko, Phys. Rev. B 82, 201408 (2010).

${ }^{27}$ V. Cvetkovic, R. E. Throckmorton, and O. Vafek, Phys. Rev. B 86, 075467 (2012)

${ }^{28}$ F. Zhang and A. H. MacDonald, Phys. Rev. Lett. 108, 186804 (2012); F. Zhang, H. Min, and A. H. MacDonald, Phys. Rev. B 86, 155128 (2012); A. H. MacDonald, J. Jung, and F. Zhang, Phys. Scr. T 146, 014012 (2012).

${ }^{29}$ T. O. Wehling, E. Şaşığlu, C. Friedrich, A. I. Lichtenstein, M. I. Katsnelson, and S. Blügel, Phys. Rev. Lett. 106, 236805 (2011).

${ }^{30}$ H. Lee, Y.-W. Son, N. Park, S. Han, and J. Yu, Phys. Rev. B 72, 174431 (2005)

${ }^{31}$ Y.-W. Son, M. L. Cohen, and S. G. Louie, Phys. Rev. Lett. 97, 216803 (2006)

${ }^{32}$ M. Otani, M. Koshino, Y. Takagi, and S. Okada, Phys. Rev. B 81, 161403(R) (2010).

${ }^{33}$ M. Otani, Y. Takagi, M. Koshino, and S. Okada, Appl. Phys. Lett. 96, 242504 (2010).

${ }^{34}$ X. Gonze et al., Comput. Phys. Commun. 180, 2582 (2009).

${ }^{35}$ F. Zhang, B. Sahu, H. Min, and A. H. MacDonald, Phys. Rev. B 82, 035409 (2010).

${ }^{36}$ S. Latil and L. Henrard, Phys. Rev. Lett. 97, 036803 (2006).

${ }^{37}$ E. McCann and V. I. Fal'ko, Phys. Rev. Lett. 96, 086805 (2006).

${ }^{38}$ K. Kechedzhi, V. I. Fal'ko, E. McCann, and B. L. Altshuler, Phys. Rev. Lett. 98, 176806 (2007).

${ }^{39}$ J. Cserti, A. Csordás, and G. Dávid, Phys. Rev. Lett. 99, 066802 (2007).

${ }^{40}$ F. L. Hirshfeld, Theor. Chem. Acta 44, 129 (1977).

${ }^{41}$ J. Yuan, J.-H. Gao, D.-H. Xu, H. Wang, Y. Zhou, and F.-C. Zhang, arXiv:1302.7123.

${ }^{42}$ D.-H. Xu, J. Yuan, Z.-J. Yao, Y. Zhou, J.-H. Gao, and F.-C. Zhang, Phys. Rev. B 86, 201404(R) (2012).

${ }^{43}$ H. Wang, J.-H. Gao, and F.-C. Zhang, Phys. Rev. B 87, 155116 (2013). 